\title{
REMARKS CONCERNING THE VARIATION OF THE LENGTH OF A CURVE.
}

BY PROFESSOR T. J. I'A. BROMWICH.

(Extract from a letter to Professor Oskar Bolza.)

Is reading through your treatment* of the shortest curve on a given surface it occurred to me that you might like to see the following investigation of the (weak) variation of the length of a curve on a surface:

As usual

$$
\begin{aligned}
& \delta \int_{t_{0}}^{t_{1}} \sqrt{x^{\prime 2}+y^{\prime 2}+z^{\prime 2}} d t=\left[\frac{x^{\prime}}{s^{\prime}} \delta x+\frac{y^{\prime}}{s^{\prime}} \delta y+\frac{z^{\prime}}{s^{\prime}} \delta z\right]_{t_{0}}^{t_{1}} \\
&-\int_{t_{0}}^{t_{1}} \sum\left[\delta x \frac{d}{d t}\left(\frac{x^{\prime}}{s^{\prime}}\right)\right] d t
\end{aligned}
$$

and the last integral may be written

$$
\int_{s_{0}}^{s_{1}} \sum\left(\delta x \frac{d^{2} x}{d s^{2}}\right) d s
$$

(from which of course your equations (32) page 130 follow). Now if $\kappa$ is the curvature of the curve, and if $l, m, n$ are the direction cosines of its principal normal,

$$
\frac{d^{2} x}{d s^{2}}=\kappa l, \text { etc. }
$$

Thus the last expression is equal to

$$
\int_{s_{0}}^{s_{1}} \delta \nu \cdot \kappa \cos \theta \cdot d s
$$

where $\delta \nu$ is the component of $\delta x, \delta y, \delta z$ resolved in a direction $\dagger$ in the tangent plane perpendicular to the tangent to the curve, while $\theta$ is the angle between the direction of $\delta \nu$ and the principal normal. Now by the elementary theory of geodesic curva-

* Bolza, Lectures on the Calculus of Variations, pp. 128-130.

$\dagger$ The vector $\delta v$ must be drawn to the left of the positive direction of the tangent, as we look down upon the tangent plane from the positive direction of the normal to the surface. 
ture, $\kappa \cos \theta$ is $\kappa_{g}$, the geodesic curvature of the curve. Thus *

$$
\delta \int_{t_{0}}^{t_{1}} \sqrt{{x^{\prime 2}+y^{\prime 2}+z^{\prime 2}}^{2}} t t=\delta \tau_{1}-\delta \tau_{0}-\int_{s_{0}}^{s_{1}} \delta \nu \cdot \kappa_{g} \cdot d s
$$

if $\delta \tau_{1}, \delta \tau_{0}$ are the components of $\delta x, \delta y, \delta z$ in the directions of the tangents at the ends of the curve.

The above process gives a very simple treatment of your investigation in the Mathematische Annalen, volume 57, page 48. For, with a closed curve, the variation of the area is evidently $\int \delta \nu \cdot d s$; and so by Euler's rule we get

or

$$
\int \delta \nu\left(1-\lambda \cdot \kappa_{g}\right) d s=0
$$

$$
\kappa_{g}=1 / \lambda .
$$

Incidentally, it would appear that the calculus of variations is almost the easiest way of finding the value of $\kappa_{g}$ in terms of Gauss's coördinates $E, F, G, u, v$, by comparing the formula (1) above with the result of varying

$$
\begin{gathered}
\int_{t_{0}}^{t_{1}} \sqrt{E u^{\prime 2}+2 F u^{\prime} v^{\prime}+G v^{\prime 2}} d t . \\
\text { QUeEN's College, Galway, IRELAND. } \\
\text { February } 23,1905 .
\end{gathered}
$$

\section{QUATERNIONS.}

A Manual of Quaternions. By Charles Jasper Joly. London, Macmillan and Co., 1905. 8 vo. xxvii +320 pages.

A QUATERnion, including a scalar and a vector as particular quaternions, is a number. This incontestable fact has not always been remembered in the discussions relating to quaternions, vector analysis, and related topics. The familiar history of the discovery and development of quaternions shows that Hamilton always kept this fact in mind, and never permitted the double use of the word vector to indicate a particular (right) quaternion, and its geometric representation, to confuse his

* The formula (1) is given in Darboux's Théorie des Surfaces, vol. 3, p. 105 , but is there derived by entirely different methods. 